\title{
Standardization of the energy performance of photovoltaic modules in real operating conditions
}

\author{
Davide Viganó ${ }^{1,2, a}$, Robert P. Kenny ${ }^{1}$, Harald Müllejans ${ }^{1}$ and Gianluca Alimonti ${ }^{2}$ \\ ${ }^{1}$ European Commission, Joint Research Centre, IET, ESTI, TP 450, 21027 Ispra (VA), Italy \\ 2 INFN and Universita' degli Studi di Milano, via Celoria 16, 20133 Milano, Italy
}

\begin{abstract}
The performance of a PV module at STC [1] is a useful indicator for comparing the peak performance of different module types, but on its own is not sufficient to accurately predict how much energy a module will deliver in the field when subjected to a wide range of real operating conditions [2]. An Energy Rating approach has to be preferred for that aim. It is currently under development the standard series IEC 61853 on Energy Rating, for which only part 1 [3] has been issued. It describes methods to characterize the module performance as a function of irradiance and temperature. The reproducibility of the power matrix measurements obtained by the three different methods specified in the standard, namely: under natural sunlight using a tracking system; under natural sunlight without tracker; and a large area pulsed solar simulator of Class AAA were evaluated and discussed $[4,5]$. The work here presented is focused on the second method listed above, which explores the real working conditions for a PV device and therefore it represents the situation where Energy Rating procedures are expected to give the largest deviations from the STC predictions. The system for continuous monitoring of module performances, already implemented at ESTI, has been recently replaced with a new system having a number of improvements described in the following. The two system results have been compared showing a discrete compatibility. The two power matrices are then merged together using a weighted average and compared to those acquired with the other two remaining "ideal" systems. An interesting tendency seems to come up from this comparison, making the power rating under real operating conditions an essential procedure for energy rating purposes.
\end{abstract}

\section{Introduction}

Photovoltaic (PV) modules were first used for terrestrial applications in the 1970s. In the intervening decades order of magnitude improvements in performance have been made together with even greater reductions in cost, through improved manufacturing processes and introduction of new materials. Of great importance to these developments and for the commercial acceptance of PV have been the introduction of standards for the performance testing and reliability of PV modules.

\footnotetext{
${ }^{a}$ Corresponding author: davide.vigano@studenti.unimi.it
}

This is an Open Access article distributed under the terms of the Creative Commons Attribution License 4.0, which permits unrestricted use, distribution, and reproduction in any medium, provided the original work is properly cited. 
Manufacturers of PV modules currently provide a power rating (Pmax) at Standard Test Conditions (STC) according to IEC 61215 [1]. These conditions correspond to an irradiance level of $1000 \mathrm{Wm}^{-2}$ at defined spectral irradiance distribution (AM1.5) and a module temperature of $25^{\circ} \mathrm{C}$ [6].

This procedure provides basic information on the maximum power developed at specified conditions; but in fact, the aim of a PV installation is to produce the largest possible amount of energy.

The usual computation of the PV energy yield is based on the multiplication of one constant value for power (e.g. at STC) by the relevant time interval. This procedure is a good approximation for those devices that show an almost-linear dependence on irradiance, like crystallineSi modules (and seldom thin-film ones). However, nowadays it's becoming more and more frequent to have new active materials available on the PV market and among them many are far away from behaving linearly.

Therefore a better estimate of the produced power should be accomplished. This is the spirit of an energy rating study, which is based on a more detailed collection of data from module measurements: the maximum power $\left(\mathrm{P}_{\max }\right)$ is measured at different temperatures $(\mathrm{T})$ and irradiance levels $(\mathrm{G})$, artificially simulating the variety of weather conditions that the PV module could encounter during its life.

Accordingly, the energy rating is given by the following sum:

$$
E=\sum_{g, k} P_{\max }(g, k) \Delta t(g, k)
$$

where the indexes $g$ and $k$ run over the irradiance range $(100,200,400,600,800,1000,1100) \mathrm{W} / \mathrm{m}^{2}$ and the temperature range $(15,25,50,75){ }^{\circ} \mathrm{C}$, according to the IEC 61853-1 standard [3].

Within this frame, the STC measurement is just a specific case in which only one term of the sum remains, giving a rough estimate of the energy actually produced.

In fact, a better estimate of the integral is a tight recording of $\mathrm{P}_{\max }$ (first factor in the product) together with an evaluation of the time intervals for which those conditions are fulfilled [7]. The latter criteria will be reported into the part 4 of IEC 61853 standard still under development.

The assessment of the advantages of an energy rating approach with respect to the STC measurement in foreseeing the amount of energy actually produced by a PV device is beyond the scope of this paper. The work here proposed is more focused on the applicability of power rating methodologies, complying where possible with IEC 61853-1 [3] and discussing the issues related to its experimental fulfillment with particular attention to the procedure "under natural sunlight without tracker".

\section{Experimental procedures}

The energy rating procedure already adopted at ESTI [2] employs systematically two of the methods envisaged by IEC 618531 [3]: natural sunlight without tracker and a Large Area Pulsed Solar Simulator.

For what concerns the outdoor field, many data were already available, acquired by an existing setup for long term measurements; however a new upgraded energy rating system (called "new-ENRA") has been recently installed at ESTI $[4,5]$.

Only the latter method is here described in detail whilst the other two "ideal" methods (indoor and outdoor with tracker) are widely described elsewhere [4, 5].

\subsection{Procedure under natural sunlight without tracker}

The longterm outdoor measurements ("ENRA" in the following) provide information on modules in real conditions (angleof-incidence effects, wind effect on module's temperature and spectral effects). These measurements were carried out on a fixed southfacing rack $45^{\circ}$ tilted (located at $45^{\circ} 48^{\prime} 42.1^{\prime \prime} \mathrm{N}$; $\left.008^{\circ} 37^{\prime} 36.3^{\prime \prime} \mathrm{E}\right)$.

According to this method, the module is naturally subject to most of the $(\mathrm{G}, \mathrm{T})$ conditions during the long exposition period. Hence, those pairs $(\mathrm{G}, \mathrm{T})$ naturally related to each other like (high G-high $\mathrm{T}$ ) and 
(low G-low T) are expected to represent the most of the collected data compared to the opposite pairs in the matrix. This is indeed the case, as shown in (Sects. 4.2 and 4.3).

Effectively, the I-V curves characteristic of the modules under test are daily determined every 5 minutes from 7:00 (UTC+1) to 19:00 (UTC+1). These data are then recorded together with ambient data.

The environmental observable quantities taken into account are: inplane $\left(45^{\circ}\right)$ irradiance, module and ambient temperatures, wind speed and wind direction. They are necessary for the verification of the environmental constraints envisaged by the IEC 61853-1 standard [3] and to perform further corrections, if required.

Irradiance is measured using two devices which have completely different spectral responses: an ESTI Sensor [8], which is basically a c-Si cell, and a pyranometer.

Both ambient and module temperature are measured by PT100 probes: one is housed in a special frame for ambient temperature, whilst two raw sensors are stuck with aluminum tape at opposite borders onto the back of the module and the average value of their readings is then used.

The wind speed and direction are determined using an ultrasound anemometer with analog outputs.

Acquisition and recording of all these experimental data are managed by a system based on PC routines developed by ESTI staff.

The recently installed "new-ENRA" setup is highly improved with respect to the previous one [5]. The new system has been working for seven months, providing a discrete amount of data: in this paper they are compared to those obtained by the "old-ENRA" records which instead cover the whole year 2011. After that the compatibility between these two sets of data is verified (Sect. 4.1) they are merged together.

A total of nearly 40'000 I-V curves measured for each of the modules under test was already available: this huge amount of data is filtered using an appropriate software which carries out a selection based on a "binning process" [9].

According to this method, each of the I-V curves measured in the outdoor field was accounted for only when its corresponding operating conditions deviated less than $\pm 2 \%$ for irradiance levels and $\pm 1^{\circ} \mathrm{C}$ for temperature values from those applied to indoor measurements. As a result of this selective procedure, only the I-V curves that fulfill both imposed requirements contribute to the average maximum power chosen to represent the $\mathrm{P}_{\max }$ output for the discrete values of irradiance and temperature.

It is important to remark that no correction is applied to the selected data, therefore taking into account only the module's characteristics under real working conditions. The comparison between this procedure and the other two "ideal" methods (Sects. 4.2 and 4.3) represents one of the key points of the present work.

\subsection{Procedure with a solar simulator}

The ESTI laboratory is equipped also with two Large Area Pulsed Solar Simulators (LAPSS) class AAA for indoor determination of the module's I-V characteristic. The older solar simulator (here referred to as "Pasan") generates a $20 \mathrm{~ms}$ flash with a characteristic irradiance decay from 1000 to $100 \mathrm{~W} / \mathrm{m}^{2}$; on the contrary, the newer "PasanIIIb" is able to maintain a constant level of irradiance for a period of $10 \mathrm{~ms}$.

The usual procedure for measuring I-V curves at varying irradiances is thus different according to the simulator taken into account: on Pasan the beginning of the voltage ramp is delayed compared to the flash so that it's applied at an irradiance level selected by the delay itself; on PasanIIIb special calibrated masks allow to achieve selected irradiance levels (listed in Sect. 1) when inserted into the lamp housing.

The software developed at ESTI to control data acquisition can automatically correct irradiance level, respecting the constraints of the standards IEC 61853-1 [3] and IEC 60891 edition 2.0. 
Both solar simulators, Pasan LAPSS and PasanIIIb use two different methods of tuning irradiance, but neither of them can be applied outdoors because one is based on fast variation of light intensity within a flash and the other relies on masks that can be used only with a solar simulator apparatus.

The mesh filtering method, recently introduced at ESTI $[4,5]$ for artificially varying the level of irradiance under natural sunlight with tracker, has been adopted also indoor to have a set of data directly comparable with the outdoor one.

\subsection{Procedure under natural sunlight with tracker}

For adjusting the irradiance under natural sunlight with tracker (simply referred to as "outdoor" in the following) a mesh filtering method is preferred to that based on the control of the angle of incidence.

According to IEC 61853-1 [1], the un-calibrated mesh filters method foresees that an appropriate reference cell is mounted close to the device under test ("DUT" in the following) to measure the incoming irradiance.

A study on the spectral modifications introduced by the mesh filters was carried out [4]; the results highlighted a sensitive effect (as large as $2.4 \%$ in $\mathrm{P}_{\max }$ ) only for CdTe at $100 \mathrm{~W} / \mathrm{m}^{2}$, while it was negligible for the other technologies under test $(<0.5 \%)$. This enhanced effect was mainly due to the big differences in spectral response between the CdTe module and the cSi reference cell. Indeed, this issue was eliminated by using a spectrally matched GaAs cell as reference in CdTe outdoor measurements.

\section{Analysis procedure}

A number of corrections are applied to the raw data in order to fit the levels of irradiance established by IEC 61853-1 [3] and to account for spectral mismatch effects. These corrections however refer only to data acquired indoor and outdoor (with tracker), but not to the outdoor field measurements: the "binning" procedure [9] adopted in that case has already been described in the previous section (Sect. 2.1).

Even if this paper reports some power matrices determined with "ideal" methods, it is more focused on the long-term performance measurement of modules in real working conditions (ENRA). Therefore, for brevity reasons, we don't report here a detailed description about the correction methods but we refer to the literature $[4,5]$.

\section{Results}

In this section the results of our experimental activity (Sect. 2) are visualized in tables.

The first paragraph (Sect. 4.1) collects the results coming from the binning procedure applied both to the data from old-ENRA (measured from January to November 2011) and new-ENRA system (from March to September 2012). After a compatibility test between these two datasets, they are merged together by means of a weighted average to provide a more reliable power rating for poly-Si and CIS in real operating conditions.

The following paragraphs (Sects. 4.2 and 4.3) contain a comparison among the power matrices measured under "real conditions" with those measured indoor and outdoor (with tracker) to evaluate the importance of wind, spectral, angle, soiling, etc... effects with respect to the "ideal conditions".

\subsection{ENRA outdoor results for poly-Si and CIS}

It is rather interesting to establish whether the power matrices obtained by the two systems described (Sect. 2.1) are compatible and -if so- which is the degree of agreement. This can be done by computing the discrepancy between the two matrices (point-by-point) and comparing it to the standard deviation that comes from the error propagation in calculating the discrepancy (which is basically a distance 
Tables 1 and 2. Matrix of t-values coming from the discrepancies expressed in std.dev. units of the two sets of data: new-ENRA-'12, old-ENRA-'11 relative to poly-Si and CIS (on the right hand side).

\begin{tabular}{|c|c|c|c|c|c|c|}
\hline \multirow{2}{*}{\multicolumn{2}{|c|}{$t=D / \sigma_{D}$}} & \multicolumn{5}{|c|}{ Temperature $\left({ }^{\circ} \mathbf{C}\right)$} \\
\hline & & 25 & 35 & 45 & 55 & 65 \\
\hline & 100 & -- & -- & -- & -- & -- \\
\hline & 200 & 0.4 & 0.6 & -- & -- & -- \\
\hline & 400 & 1.0 & 0.1 & 1.6 & 2.2 & -- \\
\hline & 600 & 1.3 & 1.5 & 0.2 & 4.1 & -- \\
\hline & 800 & 2.5 & 0.7 & 0.8 & 2.9 & -- \\
\hline & 1000 & -- & -- & 0.8 & 1.5 & -- \\
\hline
\end{tabular}

\begin{tabular}{|c|c|c|c|c|c|c|}
\hline \multirow{2}{*}{\multicolumn{2}{|c|}{$t=D / \sigma_{D}$}} & \multicolumn{5}{|c|}{ Temperature $\left({ }^{\circ} \mathbf{C}\right)$} \\
\hline & & 25 & 35 & 45 & 55 & 65 \\
\hline \multirow{6}{*}{ 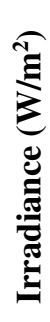 } & 100 & 0.0 & 0.2 & -- & -- & -- \\
\hline & 200 & 1.1 & 0.7 & -- & -- & -- \\
\hline & 400 & -- & 1.3 & 0.6 & -- & -- \\
\hline & 600 & -- & 2.0 & 1.6 & -- & -- \\
\hline & 800 & -- & 1.8 & 2.3 & 1.7 & -- \\
\hline & 1000 & -- & -- & 0.3 & 1.8 & -- \\
\hline
\end{tabular}

between 2 points). The square root of the quadratic sum of the standard deviation gives the final uncertainty.

The discrepancy is therefore expressed in terms of its standard deviation according to:

$$
t=\frac{D\left(x_{1}, x_{2}\right)}{\sigma_{\mathrm{D}}}=\frac{\left|x_{1}-x_{2}\right|}{\sqrt{\sigma_{1}^{2}+\sigma_{2}^{2}}} .
$$

If the discrepancy results to be within one $\sigma_{\mathrm{D}}$ (i.e. $\mathrm{t}<1$ ), it corresponds to a Confidence Level $\geq 32 \%$ $(100 \%-68 \%)$ which means that there is good accordance.

Whilst if $1 \leq \mathrm{t}<2$, then $\mathrm{CL} \geq 5 \%$ which means that there is an acceptable accordance. For larger values of $\mathrm{t}$ we would say that the accordance is poor, or that the data are not compatible.

The analysis performed on poly-Si data shows a high compatibility for 7 points over 16 . In other words, for the $44 \%$ of the points (containing data) the accordance is good. Whilst for the $75 \%(12 / 16)$ of the available data the accordance is at least acceptable.

Computing the mean discrepancy it results roughly equal to 1.38 times $\sigma_{\mathrm{D}}$ corresponding to a $\mathrm{CL} \geq 16.76 \%$.

The compatibility test carried out on CIS data has given the following results:

- $38 \%$ of points (containing data) are within $\sigma_{\mathrm{D}}$

- $92 \%$ of points (containing data) are within $2 \sigma_{\mathrm{D}}$

- Mean discrepancy $=1.18 \sigma_{\mathrm{D}} \rightarrow \mathrm{CL} \geq 23.8 \%$.

This result means that for the CIS module the repeatability is better than for crystalline. This is a quite surprising result if we consider that generally the performances of thin film devices are not stable due to the light soaking effect [10]. In fact, the module qualification procedure for thin film requires that the modules are preconditioned before any measurement [11].

On the outdoor long-term measurements, the light-soaking stability cannot be guaranteed, but this approach represents a more realistic scenario for the correct energy production estimate.

The good reproducibility observed for the CIS module is also biased from the fact that only few points of the power matrix corresponding to the most common G,T conditions are naturally "experienced" by that devices. 
Tables 3 and 4. Matrices of Pmax values and their std.dev. coming from the weighted average of the two sets of data: new-ENRA-'12, old-ENRA-'11 relative to poly-Si.

\begin{tabular}{|c|c|c|c|c|c|c|}
\hline \multirow{2}{*}{\multicolumn{2}{|c|}{$<\mathbf{P}_{\max }(\mathbf{W})>$}} & \multicolumn{5}{|c|}{ Temperature $\left({ }^{\circ} \mathrm{C}\right)$} \\
\hline & & \multirow{2}{*}{$\frac{25}{--}$} & \multirow{2}{*}{$\frac{35}{--}$} & \multirow{2}{*}{$\begin{array}{c}45 \\
--\end{array}$} & \multirow{2}{*}{$\begin{array}{l}55 \\
--\end{array}$} & \multirow{2}{*}{$\begin{array}{r}65 \\
--\end{array}$} \\
\hline & 100 & & & & & \\
\hline 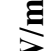 & 200 & 10.29 & 9.82 & -- & -- & -- \\
\hline 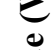 & 400 & 21.45 & 20.64 & 18.79 & 18.45 & -- \\
\hline .્. & 600 & 32.45 & 31.63 & 29.95 & 28.15 & -- \\
\hline$=$ & 800 & 40.78 & 41.07 & 39.07 & 36.99 & -- \\
\hline$=$ & 1000 & -- & -- & 47.98 & 46.00 & -- \\
\hline
\end{tabular}

\begin{tabular}{|c|c|c|c|c|c|c|}
\hline \multirow{2}{*}{\multicolumn{2}{|c|}{$\sigma_{\text {Pmax }}(W)$}} & \multicolumn{5}{|c|}{ Temperature $\left({ }^{\circ} \mathrm{C}\right)$} \\
\hline & & 25 & 35 & 45 & 55 & 65 \\
\hline \multirow{6}{*}{ 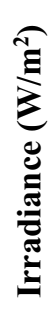 } & 100 & -- & -- & -- & -- & -- \\
\hline & 200 & 0.22 & 0.15 & -- & -- & -- \\
\hline & 400 & 0.35 & 0.37 & 0.13 & 0.40 & -- \\
\hline & 600 & 0.43 & 0.27 & 0.50 & 0.27 & -- \\
\hline & 800 & 0.50 & 0.56 & 0.61 & 0.34 & -- \\
\hline & 1000 & -- & -- & 0.68 & 0.93 & -- \\
\hline
\end{tabular}

The discrete compatibility between old-ENRA and new-ENRA data allows us to carry out a better estimate of the performances under real operating conditions by means of a weighted average:

$$
\bar{x}_{\text {weighted }}=\frac{\frac{1}{\sigma_{1}^{2}} \cdot x_{1}+\frac{1}{\sigma_{2}^{2}} \cdot x_{2}}{\frac{1}{\sigma_{1}^{2}}+\frac{1}{\sigma_{2}^{2}}} .
$$

The weighted averaged Pmax values for poly-Si are reported Table 3. Together with the associated standard deviations, they are compared to the indoor ones in Sect. 4.2 and with outdoors in Sect. 4.3.

As expected, the power matrices coming from the binning process described in Sect. 2.1 present some gaps at points corresponding to high irradiance and low temperature and vice-versa. Cells near the main diagonal of the matrix are obviously more "populated" being located at compatible pairs $(\mathrm{G}, \mathrm{T})$. Clearly the average value relative to each cell is to be considered more reliable if coming from many measurements. Sometimes, however, the standard deviation resulted larger in these cases than for cells where few points survived after the binning selection.

The observed Pmax dependence with $\mathrm{G}$ and $\mathrm{T}$ is the expected one: a smooth decrease for increasing temperature and a direct proportionality with the irradiance level.

\subsection{The importance of the "real" conditions: ENRA Vs indoor power rating}

The results obtained under real operating conditions are now compared with two different power rating procedures namely under natural sunlight (Sect. 4.5) with tracker and with a solar simulator.

The latter techniques are considered "ideal" because not affected by soiling and angle effect. Indeed, before testing the modules, they were cleaned and mounted normal to the (sun or lamp's) light beam within $\pm 2^{\circ}$ as required by the standard IEC 60904-1 [6]. The spectral effect still affects the measurements but its contribution was corrected by accounting for the spectral mismatch as stated in standards IEC 60904-3,7 [12, 13].

Now, we can assume that the indoor power matrix uncertainties are reasonably smaller than the typical standard deviations we obtained with ENRA system. Hence in the quadratic sum we obtain:

$$
\sigma_{D}=\sqrt{\sigma_{E N R A}^{2}+\sigma_{\text {indoor }}^{2}} \approx \sigma_{E N R A}
$$

To assess the compatibility between the measurements, the difference $\mathrm{D}=P_{\max }^{E N R A}-P_{\max }^{\text {indoor }}$ is computed and expressed in terms of $\sigma_{\mathrm{D}} \approx \sigma_{E N R A}$. Therefore a positive value of $\mathrm{D}$ means that the Pmax obtained with ENRA system is larger than that obtained with the solar simulator (and vice-versa). 
Tables 5 and 6. Matrices of discrepancies between ENRA and indoor Pmax measurements relative to poly-Si and CIS expressed in ENRA's std.dev. units.

\begin{tabular}{|c|c|c|c|c|c|c|}
\hline \multirow{2}{*}{\multicolumn{2}{|c|}{$\frac{P_{\max }^{E N R A}-P_{\max }^{\text {indoor }}}{\sigma_{D}}$}} & \multicolumn{5}{|c|}{ Temperature $\left({ }^{\circ} \mathbf{C}\right)$} \\
\hline & & 25 & 35 & 45 & 55 & 65 \\
\hline \multirow{6}{*}{ 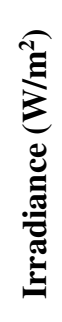 } & 100 & -- & -- & -- & -- & -- \\
\hline & 200 & 0.51 & -0.29 & -- & -- & -- \\
\hline & 400 & 0.01 & $\underline{1.10}$ & -4.17 & 0.32 & -- \\
\hline & 600 & 0.12 & 2.94 & $\underline{1.14}$ & 0.93 & -- \\
\hline & 800 & -5.01 & 0.06 & 0.43 & -0.75 & -- \\
\hline & 1000 & -- & -- & -0.63 & -0.06 & -- \\
\hline
\end{tabular}

\begin{tabular}{|c|c|c|c|c|c|c|}
\hline \multirow{2}{*}{\multicolumn{2}{|c|}{$\frac{P_{\max }^{E N R A}-P_{\max }^{\text {indoor }}}{\sigma_{D}}$}} & \multicolumn{5}{|c|}{ Temperature $\left({ }^{\circ} \mathbf{C}\right)$} \\
\hline & & 25 & 35 & 45 & 55 & 65 \\
\hline \multirow{6}{*}{ 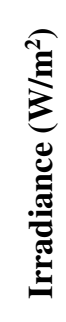 } & 100 & 7.27 & 20.37 & -- & -- & -- \\
\hline & 200 & 6.39 & 3.62 & -- & -- & -- \\
\hline & 400 & -- & 2.83 & 1.60 & -- & -- \\
\hline & 600 & -- & -0.36 & 0.08 & -- & -- \\
\hline & 800 & -- & $\underline{1.56}$ & -0.60 & -0.69 & -- \\
\hline & 1000 & -- & -- & -2.04 & -1.61 & -- \\
\hline
\end{tabular}

Tables 7 and 8. Matrices of discrepancies between ENRA and outdoor Pmax measurements relative to poly-Si and CIS expressed in ENRA's std.dev. units.

\begin{tabular}{|c|c|c|c|c|c|c|}
\hline \multirow{2}{*}{\multicolumn{2}{|c|}{$\frac{P_{\max }^{E N R A}-P_{\max }^{\text {outdoor }}}{\sigma_{D}}$}} & \multicolumn{5}{|c|}{ Temperature $\left({ }^{\circ} \mathrm{C}\right)$} \\
\hline & & 25 & 35 & 45 & 55 & 65 \\
\hline \multirow{6}{*}{ 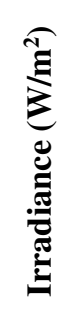 } & 100 & -- & -- & -- & -- & -- \\
\hline & 200 & 0.57 & 0.15 & -- & -- & -- \\
\hline & 400 & -0.67 & -0.49 & -5.98 & -0.34 & -- \\
\hline & 600 & -0.26 & $\underline{1.53}$ & 0.59 & -0.16 & -- \\
\hline & 800 & -5.73 & -0.98 & -0.90 & -1.52 & -- \\
\hline & 1000 & -- & -- & -2.16 & -0.55 & -- \\
\hline
\end{tabular}

\begin{tabular}{|c|c|c|c|c|c|c|}
\hline \multirow{2}{*}{\multicolumn{2}{|c|}{$\frac{P_{\max }^{E N R A}-P_{\max }^{\text {outdoor }}}{\sigma_{D}}$}} & \multicolumn{5}{|c|}{ Temperature $\left({ }^{\circ} \mathbf{C}\right)$} \\
\hline & & 25 & 35 & 45 & 55 & 65 \\
\hline \multirow{6}{*}{ 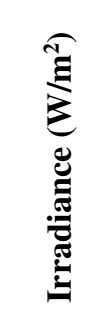 } & 100 & 5.39 & -25.02 & -- & -- & -- \\
\hline & 200 & 3.78 & 0.58 & -- & -- & -- \\
\hline & 400 & -- & $\underline{1.50}$ & -0.14 & -- & -- \\
\hline & 600 & -- & -1.16 & -1.45 & -- & -- \\
\hline & 800 & -- & 1.56 & -0.56 & -1.03 & -- \\
\hline & 1000 & -- & -- & -1.22 & -1.24 & -- \\
\hline
\end{tabular}

This comparison is carried out on both the CIS and the Si crystalline module. The results are summarized in the following tables:

The two matrices show a reasonably good agreement between indoor and ENRA power ratings: the plain text indicates data within one $\sigma_{\mathrm{D}}$. Discrepancies in the interval $\left[\sigma_{\mathrm{D}}, 2 \sigma_{\mathrm{D}}\right]$ are highlighted by underlined text; whilst deviations larger than $2 \sigma_{\mathrm{D}}$ are emphasized with a bold character and they have to be considered not consistent.

In particular, it seems that measurements at low irradiance $\left(100,200,400 \mathrm{~W} / \mathrm{m}^{2}\right)$ with ENRA system overestimate the Pmax values with respect to the indoor ones especially for CIS. This observation goes into the expected direction, confirming the influence of the non-ideality effects.

\subsection{The importance of the "real" conditions: ENRA Vs outdoor power rating}

Similarly to the previous paragraph, the measurements performed on ENRA are now compared to those coming from the outdoor data set. As in the previous case the "reference matrices" can be found elsewhere [4] and are here omitted for brevity.

The comparison results are here reported in Tables 7 and 8. 
The deviations larger than one or two- $\sigma_{D}$ are emphasized with the same criteria adopted in the previous paragraph. Again, these results show the same tendency: module's performance measured under real operating conditions (ENRA) exceeds those obtained outdoor (with tracker) which is a system still considered "ideal".

\section{Conclusions}

The Energy Rating method consists of innovative procedures to determine the energy production of a PV installation. Its advantages in terms of accuracy with respect to the more common STC method are set-off against more complex measurements which also require a specific equipment. Nevertheless, this approach is the most appropriate way to accurately predict the energy yield of non-linear PV devices.

Research is going on at ESTI improving the three procedures foreseen by the standard IEC 61853-1 [3]. The ENRA system represents module testing under real operating conditions: modules mounted on fixed racks experience all the environmental conditions. No corrections are applied to the data but they are filtered according to the described binning process (Sect. 2.1).

In this work a comparison between results obtained by the new-ENRA system and its previous version is reported. Data show a reasonably good agreement both for c-Si and CIS. The thin-film module shows a slightly better reproducibility despite of the several non-idealities such as spectral effects and light soaking which commonly affect more this kind of PV technology.

Finally, after checking their compatibility, the two matrices are merged together using a weighted average and compared to those acquired following the latter two "ideal" procedures, namely: under natural sunlight with tracker and using a solar simulator $[4,5]$. From this comparison, an interesting tendency seems to be shown for which thin-film modules performance measured under real operating conditions resulted higher than that measured in "ideal" conditions. This could be explained as the consequence of the number of non-idealities (spectral, soiling, light soaking, wind, etc. effects) which affects only the modules -and particularly the thin-film ones- under real working conditions. This evidence emphasizes the importance of the power rating procedure under natural sunlight without tracker in the energy rating framework.

\section{References}

[1] IEC 61215, edition 2.0, IEC Central Office (2005)

[2] R. P. Kenny, E. D. Dunlop, H.A. Ossenbrink, H. Müllejans, Prog. Photovolt, 14, 155-166 (2006)

[3] IEC61853-1, edition 1.0, EC Central Office (2011)

[4] R. P. Kenny, D. Viganó, E. Salis, G. Bardizza, M. Norton, H. Müllejans, W. Zaaiman, Prog. Photovolt, 21,1384-1399 (2013)

[5] D. Viganó, E. Salis, G. Bardizza, F. Perin-Gasparin, W. Zaaiman, H. Müllejans, R.P. Kenny, Proc. 27th EUPVSEC, Frankfurt, 3066-3073 (2012)

[6] IEC 60904-1, edition 2.0. IEC Central Office (2006)

[7] IEC 61853-4, edition 1.0. IEC Central Office (to be published)

[8] H. Ossenbrink, K.A. Münzer, $11^{\text {th }}$ E.C. Photovoltaic Solar Energy Conference, Montreux, (1992)

[9] K. Reitz, diploma thesis, dept. of Mech. and Automotive Eng., Univ. of Appl. Sci. Trier (2002)

[10] A. I. Chatzipanagi, R. P. Kenny, T. Sample, Prog. Photovolt. DOI:10.1002/pip.2234 (to be published)

[11] IEC 61646, edition 2.0. IEC Central Office (2008)

[12] IEC 60904-3, edition 2.0. IEC Central Office (2008)

[13] IEC 60904-7, edition 3.0. IEC Central Office (2008) 